\title{
SU(N) Coherent States
}

\author{
Manu Mathur円 and H. S. Mani [ \\ S. N. Bose National Centre for Basic Sciences \\ JD Block, Sector III, Salt Lake City, Calcutta 700098, India
}

\begin{abstract}
We generalize Schwinger boson representation of $\mathrm{SU}(2)$ algebra to $\mathrm{SU}(\mathrm{N})$ and define coherent states of $S U(N)$ using $2\left(2^{N-1}-1\right)$ bosonic harmonic oscillator creation and annihilation operators. We give an explicit construction of all (N-1) Casimirs of $\mathrm{SU}(\mathrm{N})$ in terms of these creation and annihilation operators. The $\mathrm{SU}(\mathrm{N})$ coherent states belonging to any irreducible representations of $\mathrm{SU}(\mathrm{N})$ are labelled by the eigenvalues of the Casimir operators and are characterized by (N-1) complex orthonormal vectors describing the SU(N) manifold. The coherent states provide a resolution of identity, satisfy the continuity property, and possess a variety of group theoretic properties.
\end{abstract}

PACS: $02.20 .-\mathrm{a}$

\footnotetext{
${ }^{1}$ manu@bose.res.in

${ }^{2}$ hsmani@bose.res.in
} 


\section{Introduction}

The idea of coherent state for a quantum system was realized by Schrödinger [1] way back in 1926 in the context of quantum state of classical motion for a harmonic oscillators. This simplest coherent state construction is associated with the Heisenberg-Weyl group whose Lie algebra is given in terms of a harmonic oscillator creation and annihilation operators. These states have been widely used in physics [2, 3, 4]. Later, coherent states associated with arbitrary Lie group in a given representation were constructed [5] by applying group operators to a weight vector in that particular representation. In the special case of $\mathrm{SU}(2)$ group, another equivalent way of constructing the coherent states was by exploiting the Schwinger representation of the $\mathrm{SU}(2)$ algebra [6]. This representation involves a doublet of harmonic oscillator creation annihilation operators in terms of which one generalizes the simplest Heisenberg- Weyl coherent state construction to the $\mathrm{SU}(2)$ group. In [7], using the Schwinger representation of $\mathrm{SU}(3)$ algebra, we had constructed coherent states belonging to an arbitrary representation of SU(3). The motivation of the present work is: a) to further generalise this Schwinger representation of $\mathrm{SU}(3)$ to $\mathrm{SU}(\mathrm{N})$ Lie algebra for arbitrary N, b) exploit it to construct coherent states belonging to arbitrary irreducible representations of $\mathrm{SU}(\mathrm{N})$. We also give an explicit characterization of the $\mathrm{SU}(\mathrm{N})$ coherent states in terms of (N-1) complex N-plets describing the $\mathrm{SU}(\mathrm{N})$ manifold. In the work 8], $\mathrm{SU}(\mathrm{N})$ coherent states were constructed by applying the standard procedure of applying $\mathrm{SU}(\mathrm{N})$ group operator on the highest weight state of a $\mathrm{SU}(\mathrm{N})$ representation.

The organization of the paper is as follows. In section 2 we will briefly describe the Heisenberg- 
Weyl and SU(2) coherent states in terms of harmonic oscillators. The section 2 is not only for the sake of completeness but also for setting up notation and language in a simpler setting before dealing with the larger $\mathrm{SU}(\mathrm{N})$ groups. This section also emphasize the common spirit between them and our $\mathrm{SU}(\mathrm{N})$ coherent state formalism. However, these groups being too simple, many features of larger $\mathrm{SU}(\mathrm{N})$ groups become redundant and hence this section fails to bring out a technique which can be generalized to $\mathrm{SU}(\mathrm{N})$. Therefore, in section 3, we briefly mention the $\mathrm{SU}(3)$ coherent state construction in a framework which is equivalent to the one in $\mathbb{7}$ but can be easily generalized to $\mathrm{SU}(\mathrm{N})$. In section 4 , we generalize this $\mathrm{SU}(3)$ procedure and explicitly construct $\mathrm{SU}(\mathrm{N})$ coherent states for arbitrary $\mathrm{N}$.

\section{Heisenberg-Weyl and $S U(2)$ Coherent States}

Given a group $G$ and its manifold $\mathcal{M}$, the coherent states in a given representation $R$ are functions of $q$ parameters denoted by $\left\{z^{1}, z^{2}, \ldots z^{q}\right\}$ describing $\mathcal{M}$, and are defined as

$$
\left|\vec{z}>_{R} \equiv T_{R}(g(\vec{z}))\right| 0>_{R}
$$

Here $T_{R}(g(\vec{z}))$ is a group element in the representation $R$, and $\mid 0>_{R}$ is a fixed vector belonging to $R$. In the simplest example of the Heisenberg-Weyl group, the Lie algebra contains three generators. It is defined in terms of creation annihilation operators $\left(a, a^{\dagger}\right)$ satisfying

$$
\left[a, a^{\dagger}\right]=\mathcal{I}, \quad[a, \mathcal{I}]=0, \quad\left[a^{\dagger}, \mathcal{I}\right]=0 .
$$

This algebra has only one infinite dimensional irreducible representation which can be characterized by occupation number states $\left|n>\equiv \frac{\left(a^{\dagger}\right)^{n}}{\sqrt{n !}}\right| 0>$ with $n=0,1,2 \ldots$ A generic group 
element in (11) can be characterized by $T(g)=\exp \left(i \alpha \mathcal{I}+z a^{\dagger}-\bar{z} a\right)$ with an angle $\alpha$ and a complex parameter $z$. Therefore,

$$
\begin{aligned}
\mid \alpha, z>_{\infty} & =\exp (i \alpha) \mid z>_{\infty}, \\
\mid z>_{\infty} & =\exp \left(z a^{\dagger}-\bar{z} a\right)\left|0>=\sum_{n=0}^{\infty} F_{n}(z)\right| n>,
\end{aligned}
$$

where the sum runs over all the basis vectors of the infinite dimensional representation, and

$$
F_{n}(z)=\frac{z^{n}}{\sqrt{n !}} \exp \left(-|z|^{2} / 2\right)
$$

are the coherent state expansion coefficients. This feature, i.e., an expansion of the coherent states in terms of basis vectors of a given representation with analytic functions of complex variables $\left(F_{n}(z)\right)$ as coefficients, will also be present in the case of $S U(N)$ groups. It is easy to see that Eq. (3) provides a resolution of identity with the measure $d \mu(z)=d z d \bar{z}$.

We now briefly review the next simplest example, i.e., the Schwinger representation of SU(2) Lie algebra and the associated coherent states. The Lie algebra is

$$
\left[J^{a}, J^{b}\right]=i \epsilon^{a b c} J^{c}
$$

The algebra (5) can be realized in terms of a doublet of harmonic oscillator creation and annihilation operators $a \equiv\left(a^{1}, a^{2}\right)$ and $\vec{a}^{\dagger} \equiv\left(a_{1}^{\dagger}, a_{2}^{\dagger}\right)$ respectively [6]. They satisfy the simpler bosonic commutation relation $\left[a^{i}, a_{j}^{\dagger}\right]=\delta^{i}{ }_{j}$ with $i, j=1,2$. The vacuum state is $\mid 0,0>$. In terms of these operators,

$$
Q^{a} \equiv \frac{1}{2} a_{i}^{\dagger}\left(\sigma^{a}\right)_{j}^{i} a^{j}
$$


where $\sigma^{a}$ denote the Pauli matrices. (We will generally use the convention that repeated indices are summed over). It is easy to check that the operators in (6) satisfy the $S U(2)$ Lie algebra

$$
\left[Q^{a}, Q^{b}\right]=i \epsilon^{a b c} Q^{c}
$$

We also note that

$$
\left[Q^{a}, a_{i}^{\dagger}\right]=a_{j}^{\dagger} \frac{1}{2}\left(\sigma^{a}\right)_{i}^{j}
$$

The equation (8) implies that $\left(a_{1}^{\dagger}, a_{2}^{\dagger}\right)$ transform like a SU(2) doublet. This fundamental representation of $\mathrm{SU}(2)$ will be denoted by the Young diagram $\Sigma_{[1]}$, which is a single box. Therefore, we can realize all $\mathrm{SU}(2)$ irreducible representation on the Hilbert space of harmonic oscillators created by creation operators acting on the vacuum which is a direct product of the vacuum states for $a_{1}$ and $a_{2}$. The $\mathrm{SU}(2)$ Casimir operator is given by:

$$
\mathcal{C}=\sum_{i=1}^{3} a_{i}^{\dagger} \cdot a^{i} \equiv a^{\dagger} \cdot a
$$

The eigenvalues of the Casimir $\mathcal{C}$ will be denoted by $\mathrm{C}$. The various irreducible representations of $\mathrm{SU}(2)$ are characterized by the eigenvalues of the Casimir (or occupation number operator) in (9). We also know that any irreducible representation of $\mathrm{SU}(2)$ can also be defined by its Young diagram which is obtained by arranging certain number of boxes in a row. It is easy to see that $\mathcal{C}$ just counts this number. The basis vectors of the $\mathrm{SU}(2)$ irreducible representation with $\mathcal{C}=C$ are given by:

$$
\| i_{1} i_{2} \ldots i_{C}>\equiv a_{i_{1}}^{\dagger} a_{i_{2}}^{\dagger} \ldots . . a_{i_{C}}^{\dagger} \mid 0>
$$


The dimension of the irreducible representation above is $(\mathrm{C}+1)$. With the harmonic oscillator creation and annihilation operators, $S U(2)$ coherent states can be obtained by directly generalizing (3). We define a doublet of complex numbers $\left(z^{1}, z^{2}\right)$ with the constraint:

$$
|z|^{2} \equiv\left|z^{1}\right|^{2}+\left|z^{2}\right|^{2}=1
$$

The above constraint gives 3 independent real compact parameters which define the sphere $\mathcal{M}=S^{3}$. The $S U(2)$ coherent state in the representation $C$ is now defined as

$$
\begin{aligned}
\left|z>_{C} \equiv\right| z^{1}, z^{2}>_{C} & =\exp \left(\vec{z} \cdot \vec{a}^{\dagger}\right)|0,0>|_{\vec{a}^{\dagger} \cdot \vec{a}=C} . \\
& =F^{i_{1} i_{2} \ldots i_{C}} a_{i_{1}}^{\dagger} a_{i_{2}}^{\dagger} . a_{i_{C}}^{\dagger} \mid 0> \\
& =F^{i_{1} i_{2} \ldots i_{C}} \| i_{1} i_{2} \ldots i_{C}>
\end{aligned}
$$

where,

$$
F^{i_{1} i_{2} \ldots i_{C}}=\frac{1}{C !} z^{i_{1}} z^{i_{2}} \ldots z^{i_{C}}
$$

Note that $\mathrm{F}$ in (13) are analytic functions of $z^{1}$ and $z^{2}$. Under $\mathrm{SU}(2)$ transformations:

$$
z^{i} \rightarrow z^{i}=\operatorname{expi}\left(\theta^{a} \frac{\sigma^{a}}{2}\right)^{i} z^{j}
$$

Thus the constraint (11) remains invariant under the $\mathrm{SU}(2)$ transformations (14). Therefore, the coherent states $|z>\equiv| z_{1}, z_{2}>$ transform amongst themselves on $S^{3}$. It is easy to check the resolution of identity:

$$
\int d^{2} z^{1} d^{2} z^{2} \delta\left(\left|z^{1}\right|^{2}+\left|z^{2}\right|^{2}-1\right)\left|z>_{C} C<z\right|=\frac{1}{1+C} \sum_{i_{1}, . ., i_{C}=1}^{2}\left\|i_{1} i_{2} \ldots i_{C}><i_{1} i_{2} \ldots i_{C}\right\|
$$




\section{The Schwinger $S U(3)$ Representation}

The basic technique behind the construction of $\mathrm{SU}(\mathrm{N})$ coherent states is to generalize the Schwinger representation of $\mathrm{SU}(3)$ Lie algebra [7] to $\mathrm{SU}(\mathrm{N})$ Lie algebra. We will, therefore, briefly describe the construction of SU(3) Lie algebra in terms of harmonic oscillators here in a new framework which is equivalent to that in [7] but is directly generalizable to $\mathrm{SU}(\mathrm{N})$.

The rank of the $\mathrm{SU}(3)$ group is 2 and therefore it has two fundamental representations: Any SU(3) irreducible representation can be built up from the two fundamental representations, a triplet 3 and an anti-triplet $\overline{3}$. The later is an antisymmetric combinations of the two triplets. The two fundamental representations will be associated with the two (fundamental) Young diagrams $\Sigma_{[1,0]}$, for the triplet representation and $\Sigma_{[0,1]}$, for its conjugate (anti-triplet) representation. The Young diagram $\Sigma_{[1,0]}$ is one box and $\Sigma_{[1,0]}$ is two boxes arranged vertically. We will also label the fundamental representations by the Greek indices $[\alpha],[\beta]$ taking values 1 and 2. The components of any irreducible tensor $\mathrm{T}$ transforming according to $\Sigma_{[1,0]}\left(\Sigma_{[0,1]}\right)$ will be denoted by $T[\alpha=1]_{i_{1}}\left(T[\alpha=2]_{i_{1} i_{2}} \equiv-T_{i_{1} i_{2}},\left(i_{1}, i_{2}=1,2,3\right)\right)$. ${ }^{3}$ Further, the 8 representation matrices corresponding to these two fundamental representations will be denoted by $\lambda^{a}[\alpha]$ 周. If $Q^{a}$ are the generators of $\mathrm{SU}(3)$ then under the $\mathrm{SU}(3)$ they transform as

$$
\begin{aligned}
{\left[Q^{a}, T[1]_{i_{1}}\right] } & =T[1]_{j_{1}} \lambda^{a}[1]_{i_{1}}^{j_{1}} \\
{\left[Q^{a}, T[2]_{i_{1} i_{2}}\right] } & =T[2]_{j_{1} j_{2}} \lambda^{a}[2]^{j_{1} j_{2}}{ }_{i_{1} i_{2}}
\end{aligned}
$$

The matrices on the right hand side of (16) are the matrices belonging to the fundamental

\footnotetext{
${ }^{3}$ The components of the complex conjugate of these tensors will be denoted by $T^{*}[1]^{j_{1}} T^{*}[2]^{j_{1} j_{2}}$.

${ }^{4}$ Note that $\lambda^{a}[2]^{i_{1} i_{2}}{ }_{j_{1} j_{2}} \equiv \frac{1}{2} \epsilon^{i_{1} i_{2} k} \epsilon_{j_{1} j_{2} l}\left(-\lambda^{* a}[1]\right)_{k}^{l}$.
} 
representations of $\mathrm{SU}(3)$ and satisfy the $\mathrm{SU}(3)$ Lie algebra:

$$
\begin{aligned}
& {\left[\lambda^{a}[1], \lambda^{b}[1]\right]=i f^{a b c} \lambda^{c}[1]} \\
& {\left[\lambda^{a}[2], \lambda^{b}[2]\right]=i f^{a b c} \lambda^{c}[2]}
\end{aligned}
$$

The direct product of these two tensors (representations) span the whole SU(3) representation space. Infact, any irreducible tensor can be obtained by taking direct products of $C_{1}$ of $\mathrm{T}[1]$ and $C_{2}$ of $\mathrm{T}[2]$ tensors. The corresponding Young diagram is represented by arranging $C_{2}$ of $\sigma[2]$ Young diagrams and $C_{1}$ of $\sigma[1]$ diagrams from left to right side by side. Following Schwinger representation of of $\mathrm{SU}(2)$ Lie Algebra, we introduce two sets of creation-annihilation operators $a[\alpha], a^{\dagger}[\alpha], \alpha=1,2$. The set $a[\alpha=1]$ represents three annihilation operators denoted by $a^{i_{1}}[1] \equiv\left(a[1]^{1}, a[1]^{2}, a[1]^{3}\right)$ and $a[\alpha=2]$ represents another set of three annihilation operators, $a[2]^{i_{1} i_{2}}=-a[2]^{i_{2} i_{1}}$ having three independent components $\left(a[2]^{12}, a[2]^{23}, a[2]^{31}\right)$ ]. We impose the following commutation relations on them:

$$
\begin{array}{r}
{\left[a[1]^{i_{1}}, a^{\dagger}[1]_{j_{1}}\right]=\delta_{j_{1}}^{i_{1}} \quad, \quad\left[a^{i_{1}}[1], a^{i_{2}}[1]\right]=0} \\
{\left[a[2]^{i_{1} i_{2}}, a^{\dagger}[2]_{j 1 j_{2}}\right]=\delta_{j_{1}}^{i_{1}} \delta_{j_{2}}^{i_{2}}-\delta_{j_{2}}^{i_{1}} \delta_{j_{1}}^{i_{2}} \quad, \quad\left[a[2]^{i_{1} i_{2}}, a[2]^{j 1 j_{2}}\right]=0} \\
{\left[a[1]^{i_{1}}, a[2]^{i_{2} i_{3}}\right]=0 \quad, \quad\left[a^{\dagger}[1]_{i_{1}}, a[2]^{i_{2} i_{3}}\right]=0 ;}
\end{array}
$$

We now define the $\mathrm{SU}(3)$ generators in the Hilbert space of Harmonic oscillators as:

$$
Q^{a}=a^{\dagger}[1]_{i_{1}} \lambda^{a}[1]^{i_{1}}{ }_{{ }_{2}} a[1]^{i_{2}}+\frac{1}{2 !} a^{\dagger}[2]_{i_{1} i_{2}} \lambda^{a}[2]^{i_{1} i_{2}}{ }_{j_{1} j_{2}} a[2]^{j_{1} j_{2}}
$$

Using (18), it is easy to check that

$$
\left[Q^{a}, Q^{b}\right]=i f^{a b c} Q^{c}
$$

\footnotetext{
${ }^{5}$ In [4], we had chosen $b_{1}=a[2]^{23}, b_{2}=a[2]^{31}, b_{3}=a[2]^{12}$
} 
Further,

$$
\begin{aligned}
{\left[Q^{a}, a^{\dagger}[1]_{i_{1}}\right] } & =a^{\dagger}[1]_{j_{1}} \lambda^{a}[1]^{j_{1}}{ }_{i_{1}} \\
{\left[Q^{a}, a^{\dagger}[2]_{i_{1} i_{2}}\right] } & =a^{\dagger}[2]_{j_{1} j_{2}} \lambda^{a}[2]^{j_{1} j_{2}}{ }_{i_{1} i_{2}}
\end{aligned}
$$

The equation (21) implies that $a^{\dagger}[1]$ and $a^{\dagger}[2]$ transform like $\Sigma_{[1,0]}$ and $\Sigma_{[0,1]}$ fundamental representations respectively. Therefore, all the irreducible representations of (20) can be realized on the Hilbert space created by creation operators $a^{\dagger}[1]_{i_{1}}$ and $a^{\dagger}[2]^{i_{1} i_{2}}$ acting on the vacuum state $\mid 0>$ which is direct product of vacuum states associated with each of the six harmonic oscillators. Further, the two Casimir operators of $\mathrm{SU}(3)$ in this generalization of Schwinger representation are given by:

$$
\begin{aligned}
& \mathcal{C}[1]=\sum_{i_{1}=1}^{3} a_{i_{1}}^{\dagger}[1] a^{i_{1}}[1] \\
& \mathcal{C}[2]=\sum_{i_{1}, i_{2}=1}^{3} a_{i_{1} i_{2}}^{\dagger}[2] a^{i_{1} i_{2}}[2]
\end{aligned}
$$

The eigenvalues of the two Casimirs in (22) will be denoted by $C_{1}$ and $C_{2}$ respectively.

\section{III.1 Irreducible Representations of SU(3)}

The eigenvalues of Casimirs $\left(C_{1}, C_{2}\right)$ characterize all the irreducible representations of $\mathrm{SU}(3)$. On the other hand, we can also define irreducible representations by its Young diagram which is built up by arranging certain numbers of $\Sigma_{[1,0]}$ followed by $\Sigma_{[0,1]}$ horizonally from right to left. It is easy to see that $\mathcal{C}_{1}$ and $\mathcal{C}_{2}$ just count these numbers. Therefore, we will denote a general SU(3) Young diagram by $\Sigma_{\left[C_{1}, C_{2}\right]}$ which contains $C_{1}$ of $\Sigma_{[1,0]}$ and $C_{2}$ of $\Sigma_{[2]}$ put together 
from from right to left. Therefore, a general basis vector in $\Sigma_{\left[C_{1}, C_{2}\right]}$ can be written as:

$$
\begin{aligned}
& \| i_{1}^{1} i_{1}^{2} \ldots i_{1}^{C_{2}+C_{1}} ; i_{2}^{1} i_{2}^{2} . . i_{2}^{C_{2}}>_{\Sigma_{\left[C_{1}, C_{2}\right]}} \equiv\left(e_{\left.\Sigma_{\left[C_{1}, C_{2}\right]}\right]}\right) a^{\dagger}[2]_{i_{1} i_{2}^{1}} a^{\dagger}[2]_{i_{1}^{2} i_{2}^{2}} \ldots . . a^{\dagger}[2]_{i_{1}^{C_{2}} i_{2}^{C_{2}}}[2] \\
& a^{\dagger}[1]_{i_{1} C_{2+1}} a[1]_{i_{1} C_{2}+2}^{\dagger} \ldots a^{\dagger}[1]_{i_{1}^{C_{2}+C_{1}}} \mid 0>
\end{aligned}
$$

In (23), we have characterised the basis vectors of $\Sigma_{\left[C_{1}, C_{2}\right]}$ representation by the various box indices appearing in the Young Tableau $\Sigma_{\left[C_{1}, C_{2}\right]}$. More explicitly, the index $i_{r}^{c}$ represents the index corresponding to the box appearing in the $r^{\text {th }}$ row and $c^{\text {th }}$ column. $e_{\Sigma_{\left[C_{1}, C_{2}\right]}}$ is the idempotent associated with he Young tableau $\Sigma_{\left[C_{1}, C_{2}\right]}$. In general, it is an element of group algebra corresponding to the permutation group $S\left(C_{1}+2 C_{2}\right)$. It is defined as the product of the two symmetrisers $\mathcal{S}_{1}$ (symmetrizing the indices in the first row $\left(i_{1}^{1}, i_{1}^{2}, \ldots, i_{1}^{C_{1}+C_{2}}\right), \mathcal{S}_{2}$ (symmetrizing the indices in the second row $\left(i_{2}^{1}, i_{2}^{2}, \ldots, i_{2}^{C_{1}+C_{2}}\right)$ and $C_{2}$ antisymmetrisers (acting on the $C_{2}$ columns indices $\left(\left(i_{1}^{1}, i_{2}^{1}\right),\left(i_{1}^{2}, i_{2}^{2}\right), . .,\left(i_{1}^{C_{2}}, i_{2}^{C_{2}}\right)\right)$. In fact, the idempotent for a particular Young tableau can be constructed in two different ways, first symmetrizing the indices in each row and then antisymmetrizing the indices in each column or first antisymmetrizing the indices in each column followed by symmetrizing the indices of each row. Both procedure lead to equivalent results. Here, since the column indices are already anti-symmetric, we choose the latter alternative and need to consider only the two symmetry operations along the two rows of $\Sigma_{\left[C_{1}, C_{2}\right]}$ respectively $]$ :

$$
\mathcal{S}[1]=\sum_{p \in S_{C_{2}}+C_{1}} p
$$

\footnotetext{
${ }^{6}$ In (24) and (25), the $p \in S_{n}$ is an element of the permutation group $S_{n}$ and it denotes the permutation $\left(\begin{array}{ccc}1 & 2 & \ldots . . N \\ p_{1} & p_{2} & \ldots . . p_{N}\end{array}\right)$ element of the permutation group $S_{N}$. Henceforth, we follow this notation throughout the paper.
} 


$$
\mathcal{S}[2]=\sum_{p \in S_{C_{2}}} p
$$

The idempotent is just the product of (24) and (25) and is given by:

$$
e_{\Sigma_{\left[C_{1}, C_{2}\right]}}=\mathcal{S}[1]_{[i]} \mathcal{S}[2]_{[j]}
$$

One can easily see that:

$$
\begin{aligned}
& \mathcal{C}[1]\left|i_{1}^{1} i_{1}^{2} \ldots . i_{1}^{C_{2}+C_{1}} ; i_{2}^{1} i_{2}^{2} . . i_{2}^{C_{2}}>=C_{1}\right| i_{1}^{1} i_{1}^{2} \ldots . i_{1}^{C_{2}+C_{1}} ; i_{2}^{1} i_{2}^{2} . . i_{2}^{C_{2}}> \\
& \mathcal{C}[2]\left|i_{1}^{1} i_{1}^{2} \ldots . i_{1}^{C_{2}+C_{1}} ; i_{2}^{1} i_{2}^{2} . . i_{2}^{C_{2}}>=C_{2}\right| i_{1}^{1} i_{1}^{2} \ldots . i_{1}^{C_{2}+C_{1}} ; i_{2}^{1} i_{2}^{2} . . i_{2}^{C_{2}}>
\end{aligned}
$$

Thus all the irreducible representations are eigenvectors of the two casimirs $\mathcal{C}[1]$ and $\mathcal{C}[2]$. As an example we consider $C_{1}=C_{2}=1$ corresponding to the octet representation of $\mathrm{SU}(3)$. For this representation, $\mathcal{S}[1]=\left(e+\left(i_{1}^{1}, i_{1}^{2}\right)\right)$ and $\mathcal{S}[2]=e$. Therefore, $(1,1)$ representation is given by: $\| i_{1}^{1}, i_{1}^{2} ; i_{2}^{1}>_{\Sigma_{[1,1]}}=\left[a^{\dagger}[2]_{i_{1}^{1} i_{2}^{1}} a^{\dagger}[1]_{i_{1}^{2}}+a^{\dagger}[2]_{i_{1}^{2} i_{2}^{1}} a^{\dagger}[1]_{i_{1}^{1}}\right] \mid 0>$.

In [9] similar results as in this section have been obtained by using Bargmann's techniques for $\mathrm{SU}(3)$. More recently, SU(3) multiplicity problem has been analysed by exploiting the above Schwinger construction and a mutually commuting $\operatorname{Sp}(2, \mathrm{R})$ group [10].

\section{III.2 The $S U(3)$ Coherent States}

We consider two triplets of complex numbers $z[\alpha]\left(=z^{1}[\alpha], z^{2}[\alpha], z^{3}[\alpha]\right), \alpha=1,2$ describing the Euclidean manifold $R^{6} \otimes R^{6}$. We impose the orthonormality conditions:

$$
\bar{z}[\alpha] . z[\beta] \equiv \sum_{i=1}^{3} z_{i}^{*}[\alpha] z^{i}[\beta]=\delta_{\alpha, \beta}
$$

We now define another vector $z[1,2]$ transforming as $\Sigma_{[0,1]}$ with components:

$$
z^{i_{1} i_{2}}[12] \equiv \sqrt{\frac{1}{2}}\left(z[1]^{i_{1}} z[2]^{i_{2}}-z[2]^{i_{1}} z[1]^{i_{1}}\right)=\sqrt{\frac{1}{2}} \epsilon^{\alpha_{1} \alpha_{2}} z\left[\alpha_{1}\right]^{i_{1}} z\left[\alpha_{2}\right]^{i_{2}}
$$


Using (28), we find:

$$
\begin{gathered}
z[1,2] . z[1,2]^{*} \equiv z^{i_{1} i_{2}}\left[[12] z_{i_{1} i_{2}}^{*}[12]=1\right. \\
\tilde{z}[12] . z[1] \equiv \epsilon_{i_{1} i_{2} i_{3}} z^{i_{1} i_{2}}[12] z^{i_{3}}[1]=0 \quad, \quad \tilde{z}[12] . z[2] \equiv \epsilon_{i_{1} i_{2} i_{3}} z^{i_{1} i_{2}}[12] z^{i_{3}}[2]=0
\end{gathered}
$$

We can now construct any $3 \times 3 \mathrm{SU}(3)$ matrix $\mathcal{U}_{3}$ in terms of z[1] and z[2]:

$$
\mathcal{U}_{3}=\left(\begin{array}{ccc}
z[1]_{1} & z[1]_{2} & z[1]_{3} \\
z[2]_{1} & z[2]_{2} & z[2]_{3} \\
\overline{\tilde{z}}[12]_{1} & \overline{\tilde{z}}[12]_{2} & \overline{\tilde{z}}[12]_{3}
\end{array}\right)
$$

Thus we immediately see that z[1] and z[2] with (28) and (30) completely describe the SU(3) manifold. At this stage we define the $\mathrm{SU}(3)$ coherent states generating function as:

$$
\left|z[1], z[2]>_{C_{1}, C_{2}} \equiv \exp \left(z[1]^{i_{1}} a^{\dagger}[1]_{i_{1}}+z[1,2]^{i_{1} i_{2}} a^{\dagger}[2]_{i_{1} i_{2}}\right)\right| 0>\underset{\substack{\left.\right|_{a^{\dagger}[1] . a[1]=C_{1}} \\ a^{\dagger}[2] . a[2]=C_{2}}}{ }
$$

One can see that in the expansion of (32), due to the constraints (28) and (30), each irreducible representation occurs once and only once. We will now show that (32) indeed generates all the coherent states of $\mathrm{SU}(3)$. The constraints on the right hand side of (32), $\left(a^{\dagger}[1] . a[1]=C_{1}\right.$ and $\left.a^{\dagger}[2] . a[2]=C_{2}\right)$, select different possible irreducible representations of SU(3). Note that the states in (32) are characterised by the continuous parameters $(z[1], z[2])$ on the $\mathrm{SU}(3)$ manifold. It is easy to check the $\mathrm{SU}(3)$ transformation properties of $\mathrm{z}[1]$ and $z[1,2]$,

$$
\begin{aligned}
z[1]^{i_{1}} & \rightarrow z^{\prime}[1]^{i_{1}}=\left(\operatorname{expi} \theta^{a} \lambda^{a}[1]\right)_{i_{2}}^{i_{1}} z[1]^{i_{2}} \\
z[1,2]^{i_{1} i_{2}} & \rightarrow z^{\prime}[12]^{i_{1} i_{2}}=\left(\operatorname{expi} \theta^{a} \lambda^{a}[12]\right)^{i_{1} i_{2}}{ }_{j_{1} j_{2}} z[1,2]^{j_{1} j_{2}}
\end{aligned}
$$

In (33) $\theta^{a}$ are the 8 transformation associated with the $\mathrm{SU}(3)$ group transformation. Therefore, under SU(3) transformations both z[1] and z[2] transform like a triplet and the orthonormality 
conditions (28) and (30) remain invariant under the $\mathrm{SU}(3)$ transformations and the state in (32) defined at a point $(z[1], z[2])$ transform to the coherent state at $\left(z^{\prime}[1], z^{\prime}[2]\right)$ on the $\mathrm{SU}(3)$ manifold.

From the generating function (32) we find:

$$
\left|z[1], z[2]>_{C_{1}, C_{2}}=F^{i_{1}^{1} . . i_{1}^{C_{1}+C_{2}} ; i_{2}^{1} \ldots i_{2}^{C_{2}}} a[2]_{i_{1}^{1} i_{2}^{1}}^{\dagger} . . a^{\dagger}[2]_{i_{1}^{C_{2}} i_{2}^{C_{2}}} a^{\dagger}[1]_{i_{1}^{C_{2}+1} . .} a^{\dagger}[1]_{i_{1} C_{2}+C_{1}}\right| 0>
$$

where,

$$
\begin{aligned}
C_{1} ! C_{2} ! F(z[1], z[2])^{i_{1}^{1} . . i_{1}^{C_{1}+C_{2}} ; i_{2}^{1} \ldots i_{2}^{C_{2}}}= & z[1,2]^{i_{1}^{1} i_{2}^{1}} z[1,2]^{i_{1}^{2} i_{2}^{2}} \ldots \ldots . . z[1,2]^{i_{1}^{C_{2}} i_{2}^{C_{2}}} \\
& z[1]^{i_{1}^{C_{2}+1}} z[1]^{i_{1}^{C_{2}+2}} . . z[1]^{i_{1}^{C_{2}}+C_{1}} \\
= & P_{2} z[1]^{i_{1}^{1}} z[1]^{i_{1}^{2}} \ldots z[1]^{i_{1}^{C_{2}}} z[1]^{i_{1}^{C_{2}+1}} \ldots z[1]^{i_{1}^{C_{2}}+C_{1}} \\
& z[2]^{i_{2}^{1}} z[2]^{i_{2}^{2}} \ldots z[2]^{i_{2}^{C_{2}}}
\end{aligned}
$$

In the second step in (35), we have used the anti-symmetry properties of $a^{\dagger}[2]^{\prime}$ s under the interchange of its two indices leading to $P_{2}=(2 !)^{\frac{C_{2}}{2}}$. The equation (35) clearly illustrates the following two important properties of the tensor $F(z[1], z[2])$ :

1. It is analytic function of $(z[1], z[2])$ which describe the $\mathrm{SU}(3)$ manifold,

2. It has exactly the same symmetry as that of $e_{\Sigma_{\left[C_{1}, C_{2}\right]}}$, i.e

$$
F(z[1], z[2])^{i_{1}^{1} . . i_{1}^{C_{1}+C_{2}} ; i_{2}^{1} \ldots i_{2}^{C_{2}}}=F(z[1], z[2])^{i_{1}^{1} . . i_{1}^{C_{1}+C_{2}} ; i_{2}^{1} \ldots i_{2}^{C_{2}}} e_{\Sigma_{\left[C_{1}, C_{2}\right]}}
$$

Therefore, we can write (34) as:

$$
\begin{aligned}
\mid z[1], z[2]>_{C_{1}, C_{2}} & =F^{i_{1} . . i_{C_{1}+C_{2}} j_{1} \ldots j_{C_{2}}} e_{\left.\Sigma_{\left[C_{1}, C_{2}\right.}\right]} a[2]_{i_{1} j_{1}}^{\dagger} . . a^{\dagger}[2]_{i_{C_{2}} j_{C_{2}}} a[1]_{i_{C_{2}+1}}^{\dagger} . . a[1]_{i_{C_{2}+C_{1}}}^{\dagger} \mid 0> \\
& =F^{i_{1} . . i_{C_{1}+C_{2}} j_{1} . . j_{C_{2}}} \| i_{1} j_{1}, i_{2} j_{2} . . i_{C_{2}} j_{C_{2}} i_{C_{2}+1}, i_{C_{2}+2} \ldots . i_{C_{2}+C_{1}}>_{\left[C_{1}, C_{2}\right]}
\end{aligned}
$$


All these features are similar to Heisenberg-Weyl and SU(2) Schwinger coherent states of the previous section and are discussed in detail in [7].

We now check the resolution of identity. The Haar measure on SU(3) manifold is given by:

$$
\int d \mu(z) \equiv\left(\int \prod_{\alpha=1}^{2} d z[\alpha]\right)\left(\prod_{\alpha, \beta} \delta\left(z[\alpha] . z^{*}[\beta]-\delta_{\alpha, \beta}\right)\right.
$$

We construct an operator $\mathcal{O}_{[3]}$ :

$$
\mathcal{O}_{3} \equiv \int d \mu(z)\left|z[1], z[2]>_{C_{1}, C_{2} C_{1}, C_{2}}<z[1], z[2]\right|
$$

Under SU(3) transformations (33), $\mathcal{O}_{[3]}$ remains invariant. Therefore,

$$
\left[Q^{a}, \mathcal{O}_{[3]}\right]=0, \quad \forall a=1,2, \ldots, 8
$$

The Schur's Lemma implies:

$$
\mathcal{O}_{[3]}=K I_{\left[C_{1}, C_{2}\right]}
$$

In (41), $\mathrm{K}$ is a constant and $I_{\left[C_{1}, C_{2}\right]}$ is the identity operator in the $\Sigma_{\left[C_{1}, C_{2}\right]}$ irreducible representation subspace. Thus we finally get:

$$
\begin{aligned}
& \int d \mu(z[1], z[2])\left|z[1], z[2]>_{C_{1}, C_{2} C_{1}, C_{2}}<z[1], z[2]\right|=\int d^{6} z[1] d^{6} z[2] \prod_{\alpha, \beta=1}^{2} \delta\left(z^{*}[\alpha] . z[\beta]-\delta_{\alpha, \beta}\right) \\
& \left.F^{i_{1} . . i_{C_{1}+C_{2}} j_{1} . . j_{C_{2}}} F_{k_{1} . . k_{C_{1}+C_{2}} l_{1} . . l_{C_{2}}}^{*}\right|_{i_{1} j_{1}, . . i_{C_{2}} j_{C_{2}} ; i_{C_{2}+1}, . . i_{C_{2}+C_{1}}}>_{\left[C_{1}, C_{2}\right]\left[C_{1}, C_{2}\right]}<{ }^{k_{1} l_{1}, . . k_{C_{2}} l_{C_{2}} ; k_{C_{2}+1}, . . k_{C_{2}+C_{1}} \mid} \\
& =\left.K\right|_{i_{1} j_{1}, i_{2} j_{2}, . . i_{C_{2}} j_{C_{2}} ; i_{C_{2}+1}, . . i_{C_{2}+C_{1}}}>_{\left[C_{1}, C_{2}\right]\left[C_{1}, C_{2}\right]}<{ }^{i_{1} j_{1}, i_{2} j_{2}, . . i_{C_{2}} j_{C_{2}} ; i_{C_{2}+1}, . . i_{C_{2}}+C_{1}} \mid \equiv K I_{\left[C_{1}, C_{2}\right]}
\end{aligned}
$$

\section{The Schwinger $S U(N)$ Representation}

We now generalise the ideas developed in the previous section in the case of SU(3) to the group $\mathrm{SU}(\mathrm{N})$ for arbitrary $\mathrm{N}$. The rank of the $\mathrm{SU}(\mathrm{N})$ group is $(\mathrm{N}-1)$. Therefore, there are 
(N-1) fundamental representations denoted by the Young diagrams with $1,2 \ldots$. (N-1) vertical boxes respectively. Any irreducible representation of $\mathrm{SU}(\mathrm{N})$ can be built by taking direct products of the above (N-1) fundamental representations. Following SU(2) and SU(3), we introduce $(\mathrm{N}-1)$ sets of creation annihilations operators $\left(a[1]^{i_{1}}, a^{\dagger}[1]_{i_{1}}\right) ;\left(a[2]^{i_{1} i_{2}}, a^{\dagger}[2]_{i_{1} i_{2}}\right) ; \ldots ;(a[N-$ $\left.1]^{i_{1} i_{2} \ldots . . i_{N-1}}, a^{\dagger}[N-1]_{i_{1} i_{2} \ldots . i_{N-1}}\right)$, which can be written in a compact form as $\left(a[\alpha]^{i_{1} i_{2} \ldots . . i_{\alpha}}\right.$, $\left.a^{\dagger}[\alpha]_{i_{1} i_{2} \ldots . i_{\alpha}}\right), \alpha=1,2 \ldots(N-1)$. The commutation relations are straightforward generalization of (18) and are given by:

$$
\begin{gathered}
{\left[a[\alpha]^{i_{1} i_{2} \ldots \ldots i_{\alpha}}, a^{\dagger}[\beta]_{j_{1} j_{2} \ldots \ldots j_{\beta}}\right]=\delta_{\alpha, \beta} \sum_{p \in S_{\alpha}}(-)^{|p|} \delta_{j_{p_{1}}}^{{ }^{i_{1}} \delta_{j_{p_{2}}} \ldots . . \delta_{j_{p_{\alpha}}}^{i^{i_{\alpha}}}}} \\
{\left[a[\alpha]^{i_{1} i_{2} \ldots \ldots i_{\alpha}}, a[\beta]^{j_{1} j_{2} \ldots \ldots j_{\beta}}\right]=0 \quad, \quad\left[a^{\dagger}[\alpha]_{i_{1} i_{2} \ldots \ldots i_{\alpha}}, a^{\dagger}[\beta]_{j_{1} j_{2} \ldots \ldots j_{\beta}}\right]=0 .}
\end{gathered}
$$

In (43), $|p|=0$ if $\mathrm{p}$ is an even permutation and $|p|=1$ if it is an odd permutation. We denote the (N-1) generators belonging to (N-1) fundamental representation of $\mathrm{SU}(\mathrm{N})$ by $\lambda^{a}[\alpha]$, $a=1,2, . .,\left(N^{2}-1\right)$. They satisfy the $\mathrm{SU}(\mathrm{N})$ algebra:

$$
\left[\lambda^{a}[\alpha], \lambda^{b}[\alpha]\right]=i f^{a b c} \lambda^{c}[\alpha] \quad ; \quad \alpha=1,2 \ldots(N-1)
$$

$\mathrm{In}(44) f^{a b c}$ are the $\mathrm{SU}(\mathrm{N})$ structure constants. The $\mathrm{SU}(3)$ generators in terms of Harmonic oscillators are given by:

$$
\begin{gathered}
Q^{a}=a^{\dagger}[1]_{i_{1}}[1] \lambda^{a}[1]_{i_{2}}^{i_{1}} a[1]^{i_{2}}+\frac{1}{2 !} a^{\dagger}[2]_{i_{1} i_{2}} \lambda^{a}[2]_{j_{1} j_{2}}^{i_{1} i_{2}} a[2]^{j_{1} j_{2}}+\ldots \ldots \ldots \\
\ldots .+\frac{1}{(N-1) !} a^{\dagger}[N-1]_{i_{1} i_{2} . . i_{N-1}} \lambda^{a}[N-1]_{j_{1} . . j_{N-1}}^{i_{1} . i_{N-1}} a[N-1]^{j_{1} j_{2} \ldots j_{N-1}} \\
=\sum_{\alpha=1}^{N-1} \frac{1}{\alpha !} a^{\dagger}[\alpha]_{i_{1}, i_{2} \ldots i_{\alpha}} \lambda^{a}[\alpha]_{j_{1} j_{2} \ldots j_{\alpha}}^{i_{1} i_{2} \ldots i_{\alpha}} a[\alpha]^{j_{1} j_{2} \ldots . j_{\alpha}}
\end{gathered}
$$


The commutation relations (43) imply:

$$
\left[Q^{a}, Q^{b}\right]=i f^{a b c} Q^{c}
$$

It is easy to verify that under $\mathrm{SU}(3)$ transformations the various creation operators belonging to the $\alpha^{\text {th }}$ representation transform amongst themselves as:

$$
\left[Q^{a}, a_{i_{1} i_{2} \ldots i_{\alpha}}^{\dagger}[\alpha]\right]=a_{j_{1} j_{2} \ldots j_{\alpha}}^{\dagger}[\alpha]\left(\lambda^{a}\right)_{i_{1} i_{2} \ldots i_{\alpha}}^{j_{1} j_{2} \ldots j_{\alpha}}[\alpha]
$$

This implies that all the irreducible representations of $\mathrm{SU}(\mathrm{N})$ can be realised on the Hilbert space created by the above creation operators acting on the vacuum. Further, the (N-1) Casimir

operators are just the (N-1) number operators corresponding to (N-1) types of creation annihilation operators and are given by:

$$
\begin{aligned}
\mathcal{C}[1] & =\sum_{i_{1}=1}^{N} a^{\dagger}[1]_{i_{1}} a[1]^{i_{1}} \\
\mathcal{C}[2] & =\frac{1}{2 !} \sum_{i_{1}, i_{2}=1}^{N} a^{\dagger}[2]_{i_{1} i_{2}} a[2]^{i_{1} i_{2}} \\
& \ldots \\
\mathcal{C}[N-1] & =\frac{1}{(N-1) !} \sum_{i_{1}, i_{2}, \ldots, i_{N-1}=1}^{N} a^{\dagger}[N-1]_{i_{1} i_{2} \ldots i_{N-1}} a[N-1]^{i_{1} i_{2} \ldots i_{N-1}}
\end{aligned}
$$

It is easy to check that the $(\mathrm{N}-1)$ Casimir operators in $(48)$ commute with all the $\left(N^{2}-1\right)$ $\mathrm{SU}(\mathrm{N})$ generators in (45).

\section{IV.1 The Irreducible Representations of SU(N)}

All irreducible representations of $\mathrm{SU}(\mathrm{N})$ can be constructed by taking direct products of the (N-1) fundamental representations and then applying appropriate projection operators. Like 
the $\mathrm{SU}(3)$ case in the previous section, we now consider the most general $\mathrm{SU}(\mathrm{N})$ irreducible representation $\Sigma_{\left[C_{1}, C_{2} \ldots \ldots . C_{N-1}\right]}$ containing $C_{\alpha}$ copies of the $\alpha^{\text {th }}$ fundamental representations. We formally writei]:

$$
\left|C_{1}, C_{2}, . ., C_{N-1}>\equiv e_{\Sigma_{\left[C_{1} \ldots C_{N-1}\right]}}\left(a^{\dagger}[1]\right)^{C_{1}}\left(a^{\dagger}[2]\right)^{C_{2}}\left(a^{\dagger}[3]\right)^{C_{3}} \ldots\left(a^{\dagger}[N-1]\right)^{C_{N-1}}\right| 0>
$$

Just as in the case of $\mathrm{SU}(3)$ in the previous sections, all the creation operators being completely anti-symmetric in their indices, the idempotent $e_{\left.\Sigma_{\left[C_{1} . C_{N-1}\right]}\right]}$ in (49) is constructed by multiplying all (N-1) symmetrizers:

$$
e_{\Sigma_{\left[C_{1} \ldots C_{N-1}\right]}}=\mathcal{S}_{1} \mathcal{S}_{2} \ldots \mathcal{S}_{N-1}
$$

where $\mathcal{S}_{1}, \mathcal{S}_{2} \ldots \mathcal{S}_{N-1}$ are the elements of permutation group algebras associated with the permutation groups $S_{C_{1}+. .+C_{N-1}}, S_{C_{2}+. .+C_{N-1}}, \ldots, S_{C_{N-1}}$ respectively,

$$
\begin{aligned}
\mathcal{S}_{1} & \equiv \frac{1}{\left(C_{1}+\ldots+C_{N-1}\right) !} \sum_{p \in S_{C_{1}+\ldots+C_{N-1}}} p \\
\mathcal{S}_{2} & \equiv \frac{1}{\left(C_{2}+\ldots+C_{N-1}\right) !} \sum_{p \in S_{C_{2}+\ldots+C_{N-1}} p} p \\
& \ldots \\
\mathcal{S}_{N-1} & \equiv \frac{1}{\left(C_{N-1}\right) !} \sum_{p \in S_{C_{N-1}}} p
\end{aligned}
$$

Again, as in the case of $\mathrm{SU}(3)$, it is easy to verify:

$$
\mathcal{C}_{\alpha}\left|C_{1}, C_{2}, . ., C_{N-1}>=C_{\alpha}\right| C_{1}, C_{2}, . ., C_{N-1}>
$$

Therefore, the Casimir operator $\mathcal{C}_{\alpha}$ acting on an irreducible representation generated by the basis vectors $\mathcal{C}_{\alpha} \mid C_{1}, C_{2}, . ., C_{N-1}>$ just counts the number of times the $\alpha^{\text {th }}$ fundamental

\footnotetext{
${ }^{7}$ The explicit form of these basis vectors are given at the end of the next section (64) and 65
} 
representation appears in it. This is again similar to the $\mathrm{SU}(3)$ case in the previous section. We now exploit this feature to construct the $\mathrm{SU}(\mathrm{N})$ coherent states.

\section{IV.2 The SU(N) Coherent States}

We consider $(\mathrm{N}-1) \mathrm{N}$-plets of complex numbers $z[\alpha]\left(=z^{1}[\alpha], z^{2}[\alpha], \ldots z^{N}[\alpha]\right), \alpha=1,2, . ., N-1$ describing the Eucledian manifold which is a direct product of (N-1) $R^{2 N}$ We impose the orthonormality conditions:

$$
z^{*}[\alpha] . z[\beta]=\delta_{\alpha, \beta}
$$

We now define another vector $z[1, N-1]$ with components:

$$
\begin{aligned}
z^{i_{1} i_{2} . . i_{N-1}}[1, N-1] & \equiv \sqrt{\frac{1}{(N-1) !}} \sum_{p \in S_{N-1}}(-)^{|p|}\left(z[1]^{i_{p_{1}}} z[2]^{i_{p_{2}}} \ldots z[N-1]^{i_{p_{N-1}}}\right) \\
& =\sqrt{\frac{1}{(N-1) !}} \epsilon^{\alpha_{1} \alpha_{2} \ldots \alpha_{N-1}} z\left[\alpha_{1}\right]^{i_{1}} z\left[\alpha_{2}\right]^{i_{2} \ldots . . z\left[\alpha_{N-1}\right]}
\end{aligned}
$$

Using (53), we find:

$$
\begin{array}{r}
z[1, N-1] . z[1, N-1]^{*} \equiv z^{i_{1} i_{2} . i_{N-1}}\left[[1, N-1] z^{*}{ }_{i_{1} i_{2} . . i_{N-1}}[1, N-1]=1\right. \\
\tilde{z}[1, N-1] . z[\alpha] \equiv \epsilon_{i_{1} i_{2} \ldots . i_{N}} z^{i_{1} i_{2} \ldots i_{N-1}}[1, N-1] z^{i_{N}}[\alpha]=0
\end{array}
$$

We can now construct any $N \times N \mathrm{SU}(\mathrm{N})$ matrix $\mathcal{U}_{N}$ in terms of z[1], z[2], ..., z[N-1]:

$$
\mathcal{U}_{N}=\left(\begin{array}{ccc}
z[1]^{1} & z[1]^{2} & \ldots z[1]^{N} \\
z[2]^{1} & z[2]^{2} & \ldots z[2]^{N} \\
: & & \\
: & & \\
z[N-1]^{1} & z[N-1]^{2} & \ldots . . z[N-1]^{N} \\
\overline{\tilde{z}}[1 . . N-1]^{1} & \overline{\tilde{z}}[1 . . N-1]^{2} & \overline{\tilde{z}}[1 . . N-1]^{N}
\end{array}\right)
$$

Thus we immediately see that $z[1], z[2], \ldots, z[\mathrm{~N}-1]$ with (55) describe $\mathrm{SU}(\mathrm{N})$ manifold. We will now exploit this simple characterisation of $\mathrm{SU}(\mathrm{N})$ manifold to construct coherent states 
belonging to all the irreducible representations of $\mathrm{SU}(\mathrm{N})$ group. For this purpose, we construct a new set of $(\mathrm{N}-1)$ parameters:

$$
\begin{aligned}
z[1,1]^{i_{1}} & \equiv z[1]^{i_{1}} \\
z[1,2]^{i_{1} i_{2}} & \equiv \sqrt{\frac{1}{2 !}} \sum_{p \in S_{2}}(-)^{|p|} z[1]^{i_{p_{1}}} z[2]^{i_{p_{2}}} \\
z[1,3]^{i_{1} i_{2} i_{3}} & \equiv \sqrt{\frac{1}{3 !}} \sum_{p \in S_{3}}(-)^{|p|} z[1]^{i_{p_{1}}} z[2]^{i_{p_{2}} z[3]^{i_{p_{3}}}} \\
& \cdots \\
z[1, N-1]^{i_{1} i_{2} . . i_{N-1}} & \equiv \sqrt{\frac{1}{(N-1) !}} \sum_{p \in S_{N-1}}(-)^{|p|} z[1]^{i_{p_{1}}} z[2]^{i_{p_{2}}} . . z[N-1]^{i_{p_{N-1}}}
\end{aligned}
$$

We now define the $\mathrm{SU}(\mathrm{N})$ coherent states generating function as:

$$
\begin{aligned}
& \left|z[1], z[2], . ., z[N-1]>_{C_{1}, C_{2} . . C_{N-1}} \equiv \exp \left(\sum_{\beta=1}^{N-1} z[1, \beta] \cdot a^{\dagger}[\beta]\right)\right| 0>\left.\right|_{a^{\dagger}[\alpha] . a[\alpha]=C_{\alpha}} \\
& \equiv \exp \left(z[1]^{i_{1}^{1}} a^{\dagger}[1]_{i_{1}^{1}}+z[1,2]^{i_{1}^{2} i_{2}^{2}} a^{\dagger}[2]_{i_{1} i_{2}^{2}}+\ldots \ldots\right. \\
& \left.\ldots \ldots+z[1, N-1]^{i_{1}^{N-1} i_{2}^{N-1} \ldots i_{N-1}^{N-1}} a^{\dagger}[N-1]_{i_{1}^{N-1} i_{2}^{N-1} . . i_{N-1}^{N-1}}\right)|0>|_{a^{\dagger}[\alpha] . a[\alpha]=C_{\alpha}}
\end{aligned}
$$

The constraints on the right hand side of the generating function (58) ensure that we only generate the states which are eigenstates of all the (N-1) casimir operators and thus selecting all possible irreducible representations of $\mathrm{SU}(\mathrm{N})$, i.e,

$$
\mathcal{C}[\alpha]\left|z[1], z[2], . ., z[N-1]>_{C_{1}, C_{2} . . C_{N-1}}=C[\alpha]\right| z[1], z[2], . ., z[N-1]>_{C_{1}, C_{2} . . C_{N-1}}
$$

for $\alpha=1,2 . .(N-1)$. We will now show that the states thus obtained are the coherent states. All states in (58) are defined continuously over the $\mathrm{SU}(\mathrm{N})$ manifold parametrised by $(\mathrm{z}[1], \mathrm{z}[2], \ldots, \mathrm{z}[\mathrm{N}-$ 1]). It is easy to check the $\mathrm{SU}(\mathrm{N})$ transformation properties of $z[1, \alpha], \alpha=1,2 . .(N-1)$.

$$
z[1, \alpha]^{i_{1} . . i_{\alpha}} \rightarrow z^{\prime}[1, \alpha]^{i_{1} . i_{\alpha}}=\left(\operatorname{expi} \sum_{a=1}^{N^{2}-1} \theta^{a} \lambda^{a}[\alpha]\right)_{j_{1} . . j_{\alpha}}^{i_{1} . i_{\alpha}} z[1, \alpha]^{j_{1} . . j_{\alpha}}
$$


In (60) $\theta^{a}$ are $\left(N^{2}-1\right)$ parameters describing a point on the $\mathrm{SU}(\mathrm{N})$ manifold. The equation (60) implies that all $z[\alpha](\alpha=1,2, . ., N-1)$ transform as $\mathrm{N}$-plets of SU(N), i.e,:

$$
z[\alpha] \rightarrow z^{\prime}[\alpha]=\left(\operatorname{expi} \sum_{a=1}^{N^{2}-1} \theta^{a} \lambda^{a}[1]\right)_{j_{1}}^{i_{1}} z[\alpha]^{j_{1}}
$$

We see that the orthonormality conditions (53) remain invariant under the SU(N) transformations. The coherent states defined at a point $(z[1], z[2] . ., z[1, N-1])$ transform to the coherent states at $\left(z^{\prime}[1], z^{\prime}[2], . z^{\prime}[1, N-1]\right)$ of the $\mathrm{SU}(\mathrm{N})$ manifold.

Expanding the exponential we find:

$$
\begin{gathered}
\mid z[1], . ., z[N-1]>_{C_{1}, . . C_{N-1}}=P\left[\prod_{h=1}^{h_{\max }} \prod_{v=1}^{\left(C_{h}+C_{h+1} \ldots+C_{N-1}\right)} z^{i_{h}^{v}}[h]\right]\left[\prod_{v(1)=1}^{C_{N-1}} a^{\dagger}[N-1]_{i_{1}^{v(1)} i_{2}^{v(1)} \ldots . i_{N-1}^{v(1)}}\right] \\
{\left[\prod_{v(2)=C_{N-1}+1}^{C_{N-1}+C_{N-2}} a^{\dagger}[N-2]_{i_{1}^{v(2)} i_{2}^{v(2)} \ldots i_{N-2}^{v(2)}}\right] \ldots . .\left[\prod_{v(N-1)=C_{N-1}+. .+C_{2}+1}^{C_{N-1}+\ldots+C_{1}} a^{\dagger}[1]_{i_{1}^{v(N-1)}}\right] \mid 0>}
\end{gathered}
$$

In (62), we have chosen those terms in the expansion which are eigenvectors of the Casimir operators $\mathcal{C}_{\beta}$ with eigenvalues $C_{\beta}$ and $h_{\max }$ is the maximum value of $\beta$ such that $C_{\beta} \neq 0$. $P=\left[\prod_{\alpha=1}^{N-1} P_{\alpha}\right]$ with $P_{\alpha}=(\alpha !)^{\frac{C_{\alpha}}{2}}$. We note the following important symmetry and antisymmetry properties of (62):

1. The $(z[1], z[2], \ldots z[N-1])$ dependent part of (62) has inbuilt invariance under interchange of any two indices along a row of the Young tableau $\Sigma_{\left[C_{1}, C_{2}, \ldots C_{N-1}\right]}$, i.e, it is invariant under all the symmetry operations given in (51),

$$
\left(\prod_{h=1}^{h_{\max }} \prod_{v=1}^{\left(C_{h}+C_{h+1} \ldots+C_{N-1}\right)} z^{i^{v}}[h]\right) \mathcal{S}_{\alpha}=\left(\prod_{h=1}^{h_{\max }} \prod_{v=1}^{\left(C_{h}+C_{h+1} .+C_{N-1}\right)} z^{i_{h}^{v}}[h]\right)
$$

\footnotetext{
${ }^{8}$ In (62) $h=1,2 . . h_{\max }$ counts the horizontal rows and $\mathrm{v}$ is used to count the vertical columns of $\Sigma_{\left[C_{1}, . ., C_{N-1}\right]}$. Note that it is the maximum number of horizontal rows in the Young diagram.
} 
2. The harmonic oscillator dependent part of (62) has inbuilt anti-symmetric properties along any column indices of the Young tableau $\Sigma_{\left[C_{1}, C_{2}, \ldots . C_{N-1}\right]}$.

Therefore, we can write (62) as:

$$
\begin{aligned}
& \mid z[1], . ., z[N-1]>_{C_{1}, . . C_{N-1}}=\prod_{h=1}^{h_{\max }} \prod_{v=1}^{\left(C_{h}+C_{h+1} \ldots+C_{N-1}\right)} z^{i_{h}^{v}}[h] e_{\Sigma_{\left[C_{1}, C_{2}, \ldots, C_{N-1}\right]}} \\
& \prod_{v(1)=1}^{C_{N-1}} a^{\dagger}[N-1]_{i_{1}^{v(1)} i_{2}^{v(1)} \ldots i_{N-1}^{v(1)}} \prod_{v(2)=C_{N-1}+1}^{C_{N-1}+C_{N-2}} a^{\dagger}[N-2]_{i_{1}^{v(2)} i_{2}^{v(2)} \ldots . i_{N-2}^{v(2)} . .} \\
& C_{N-1}+\ldots+C_{1} \\
& \cdots \prod_{v(N-1)=C_{N-1}+. . C_{2}+1} a^{\dagger}[1]_{i_{1}^{v(N-1)}} \mid 0>
\end{aligned}
$$

In (64), $e_{\Sigma_{\left[C_{1}, C_{2}, \ldots, C_{N-1}\right]}}$ is defined by the equation (50). Infact, these are exactly basis vectors of the irreducible representation $\Sigma_{\left[C_{1}, C_{2} \ldots \ldots ., C_{N-1}\right]}$. We denote them by:

$$
\begin{gathered}
\left.\|\left(i_{1}^{1} i_{1}^{2} \ldots \ldots . i_{1}^{C_{1}+. .+C_{N-1}}\right) ;\left(i_{2}^{1} i_{2}^{2} \ldots \ldots . i_{2}^{C_{2}+. .+C_{N-1}}\right) ; \ldots \ldots ;\left(i_{N-1}^{1} i_{N-1}^{2} \ldots \ldots . i_{N-1}^{C_{2}+. .+C_{N-1}}\right)\right\rangle_{\Sigma_{\left[C_{1}, C_{2}, \ldots, C_{N-1}\right]}} \\
\equiv e_{\Sigma_{\left[C_{1}, C_{2}, \ldots, C_{N-1}\right]}} \prod_{v(1)=1}^{C_{N-1}} a^{\dagger}[N-1]_{i_{1}^{v(1)}} i_{2}^{v^{(1)} \ldots . i_{N-1}^{v(1)}} \prod_{v(2)=C_{N-1}+1}^{C_{N-1}+C_{N-2}} a^{\dagger}[N-2]_{i_{1}^{v(2)}} j_{2}^{v(2)} \ldots i_{N-2}^{v(2)} \\
\ldots \ldots \ldots \ldots \ldots \ldots \\
{ }_{v(N-1)=C_{N-1}+. . C_{2}+1} a^{C_{N-1}+\ldots+C_{1}}[1]_{i_{1}^{v(N-1)}} \mid 0>
\end{gathered}
$$

Thus the states belonging to $\Sigma_{\left[C_{1}, . ., C_{N-1}\right]}$ in (58) irreducible representations are given by:

$$
\begin{gathered}
\mid z[1], z[2], . ., z[N-1]>_{C_{1}, C_{2} . . C_{N-1}}=\left(\prod_{h=1}^{h_{\max }} \prod_{v=1}^{\left(C_{h}+C_{h+1} \ldots+C_{N-1}\right)} z^{i_{h}^{v}}[h]\right) \\
\left.\|\left(i_{1}^{1} i_{1}^{2} \ldots \ldots i_{1}^{C_{1}+. .+C_{N-1}}\right) ;\left(i_{2}^{1} i_{2}^{2} \ldots \ldots . i_{2}^{C_{2}+. .+C_{N-1}}\right) ; \ldots \ldots . ;\left(i_{N-1}^{1} i_{N-1}^{2} \ldots \ldots . i_{N-1}^{C_{2}+. .+C_{N-1}}\right)\right\rangle_{\Sigma_{\left[C_{1}, C_{2}, \ldots, C_{N-1}\right]}}
\end{gathered}
$$

We now check the resolution of identity. This is similar to the $\mathrm{SU}(3)$ case in the previous sections. The $\mathrm{SU}(\mathrm{N})$ Haar measure is:

$$
\int d \mu(z) \equiv\left(\int \prod_{\alpha=1}^{N-1} d z[\alpha]\right)\left(\prod_{\alpha, \beta} \delta\left(z[\alpha] . z^{*}[\beta]-\delta_{\alpha, \beta}\right)\right)
$$


We construct an operator $\mathcal{O}_{[N]}$ :

$$
\mathcal{O}_{N} \equiv\left(\int d \mu(z)\left|z[1], z[2], . ., z[N-1]>_{C_{1}, C_{2} . . C_{N-1}} \quad C_{1}, C_{2} . . C_{N-1}<z[1], z[2], . ., z[N-1]\right|\right.
$$

Under $\mathrm{SU}(\mathrm{N})$ transformations $(60), \mathcal{O}_{[3]}$ remains invariant. Therefore,

$$
\left[Q^{a}, \mathcal{O}_{[N]}\right]=0, \quad \forall a=1,2, \ldots, N^{2}-1
$$

The Schur's Lemma implies:

$$
\mathcal{O}_{[N]}=K I_{\left[C_{1}, C_{2}, \ldots, C_{N-1}\right]}
$$

In (70), $\mathrm{K}$ is a constant and $I_{\left[C_{1}, . ., C_{N-1}\right]}$ is the identity operator in the $\Sigma_{\left[C_{1}, . ., C_{N-1}\right]}$ irreducible representation subspace. Therefore, the states in (66) are indeed the coherent states. We have thus constructed all the $\mathrm{SU}(\mathrm{N})$ coherent states belonging to different irreducible representations of $\mathrm{SU}(\mathrm{N})$.

\section{Summary and Discussion}

We have generalized Schwinger representation of SU(2) algebra in terms of harmonic oscillotrs to the group $\mathrm{SU}(\mathrm{N})$. We have exploited this construction to construct $\mathrm{SU}(\mathrm{N})$ coherent states and characterized $\mathrm{SU}(\mathrm{N})$ manifold in terms of complex vectors. In this sense our $\mathrm{SU}(\mathrm{N})$ coherent states definition is analogous to that of Heisenberg-Weyl coherent states. This method is quite general and can be generalized to other Lie groups and their manifolds. We feel our approach is more useful for practical calculations compared to the standard group theoretical approach and can be applied to various problems. In condensed matter physics, coherent states for the 
Lie group $S U(2)$ have been very useful for studying Heisenberg spin systems using the path integral formalism [11, 12, 13, 14]. These studies have been generalized to systems with $S U(N)$ symmetry, although such studies have usually been restricted to the completely symmetric representations [12, 16]. Therefore, our formulation can be used to write down the field theory for the $S U(N)$ Heisenberg model and study its spectrum and topological aspects as in the $S U(2)$ case [17]. Using the techniques discussed in the paper, one can also construct $\mathrm{SU}(\mathrm{N})$ coherent states with fixed values of Cartan diagonal generators. The special cases of $\mathrm{SU}(2)$ coherent states with fixed charge and $\mathrm{SU}(3)$ nonlinear coherent states with fixed charge and hypercharge were constructed in [18] and [19] respectively.

\section{Acknowledgments}

We would like to thank Prof. Binayak Dutta Roy for useful discussions. One of the authors (M.M) would also like to thank Prof. Diptiman Sen for his involvement and discussions at the initial stages of this work.

\section{References}

[1] E. Schrodinger, Naturwissenshafter, 14, 644, (1926).

[2] R. J. Glauber, Phys. Rev. 130 (1963) 2529, Phys. Rev. 131 (1963) 2766

[3] J. R. Klauder, J. Math. Phys. 4 (1963) 1055,

J. R. Klauder and B.-S. Skagerstam, Coherent States, Applications in Physics and Mathmatical Physics (World Scientific, Singapore, 1985). 
[4] E. C. G. Sudarshan, Phys. Rev. Lett. 10277.

[5] A. Perelomov, Generalized Coherent States and Their Applications (Springer-Verlag, New York, 1986); A. Perelomov, Sov. Phys. Usp. 20 (1977) 703.

[6] J. Schwinger, Atomic Energy Commission Report No. NYO-3071 (1952) or D. Mattis, The Theory of Magnetism (Harper and Row, 1982).

[7] Manu Mathur, Diptiman Sen, J. Math. Phys. 42 (2001) 4181.

[8] Kae Nemoto, J. Phys. A 33 (2000) 3493.

[9] J. S. Prakash and H. S. Sharatchandra, J. Math. Phys. 37 (1996) 6530, hep-th/9607101.

[10] S. Chaturvedi and N. Mukunda, quant-ph/0204119 and quant-ph/0204120.

[11] D. P. Arovas and A. Auerbach, Phys. Rev. B 38 (1988) 316.

[12] E. Manousakis, Rev. Mod. Phys. 63 (1991) 1; A. Auerbach, Interacting Electrons and Quantum Magnetism (Springer-Verlag, New York, 1994).

[13] E. Fradkin, Field Theories of Condensed Matter Systems (Addison-Wesley, Reading, 1991).

[14] S. Sachdev, in Low Dimensional Quantum Field Theories for Condensed Matter Physicists, edited by Y. Lu, S. Lundqvist and G. Morandi (World Scientific, Singapore, 1995), condmat/9303014.

[15] C. Brif, A. Vourdas and A. Mann, J. Phys. A 29 (1996) 5873. 
[16] D. M. Gitman and A. L. Shelepin, J. Phys. A 26 (1993) 313;

[17] F. D. M. Haldane, Phys. Rev. Lett. 61 (1988) 1029.

[18] Bhaumik D, Bhaumik K and Dutta Roy B1976, J. Phys. A 91507.

[19] Hongyi Fan, Guichuan Yu, J. Phys. A 34 (2001) 5995. 